Review Article

\section{Auditory effects and consequences of noise pollution in humans: A scoping review}

\author{
Nazia Begam ${ }^{1}$ and Abu Bashar ${ }^{2 *}$ \\ ${ }^{1}$ Specialist, M.O. ENT, ECHS Hospital, Naraingarh, Haryana, India \\ ${ }^{2} \mathrm{MD}$, Assistant Professor, Community Medicine, IMS, BHU, Varanasi, India
}

\author{
More Information \\ *Address for Correspondence: Abu Bashar, \\ MD, Assistant Professor, Community Medicine \\ IMS, BHU, Varanasi, India, \\ Email: dr.bashartheultimate86@gmail.com \\ Submitted: 18 September 2020 \\ Approved: 06 November 2020 \\ Published: 09 November 2020 \\ How to cite this article: Begam N, Bashar A. \\ Auditory effects and consequences of noise \\ pollution in humans: A scoping review. Adv Treat \\ ENT Disord. 2020; 4: 006-010. \\ DOI: 10.29328/journal.ated.1001011 \\ Copyright: () 2020 Begam N, et al. This is \\ an open access article distributed under the \\ Creative Commons Attribution License, which \\ permits unrestricted use, distribution, and \\ reproduction in any medium, provided the \\ original work is properly cited. \\ Keywords: Noise; Auditory; Non-auditory; NIHL; \\ Genes; Public health \\ A) Check for updates \\ OPen Access
}

\begin{abstract}
Noise is widespread in everyday life and can cause both auditory and non-auditory health impacts. Noise-induced hearing loss remains highly prevalent in occupational settings and is now increasingly caused by exposure to social and environmental noise. Incidence of noise-induced hearing loss ( $\mathrm{NIHL}$ ) has been observed to increase substantially in the recent years. Severa advances have taken place in past few years for understanding the molecular basis of NIHL. Ou understanding of molecular mechanisms implicated in noise-induced hair-cell and nerve damage has significantly increased. Research in the field of genetics is also advancing at a rapid speed, and several genes linked to NIHL have been discovered. This could help in developing preventive and treatment strategies. This review article focuses on the current research and future trends on auditory effects and consequences of noise pollution in humans, stressing the importance of adequate noise prevention and mitigation strategies as a public health measures.
\end{abstract}

cause receipt of many complaints to local law and order enforcing authorities. Noise is widespread in urban settings and the availability of quiet places is decreasing in these. In the European Union, about 56 million people (54\%) living in areas with more than 250000 inhabitants are exposed to road traffic noise of more than average Day Evening Night Sound Level i.e. LDEN of $55 \mathrm{~dB}$ (Decibels) per year, which is thought to be too risky for health [9]. Thus, understanding of social and environmental noise and findings ways to lessen them is very vital for public health.

Noise-Induced Hearing Loss (NIHL) can be caused by a one-time exposure to an intense impulse sound or more frequently by a long-term constant exposure with sound pressure levels (SPLs) more than 80-85 dBHL (Decibels Hearing Level). Noise pollution is now increasingly recognized as a public health problem. The World Health Organization (WHO) estimates that around $10 \%$ of the world population is currently exposed to SPLs that have the potential to cause NIHL. Road traffic noise is one of the main sources of noise pollution, particularly in urban areas. The noise levels have been monitored across different sections of the town/city, and ambient noise levels have been found to be above permissible limits.

An increased incidence of NIHL is being observed in the 
population round the globe in the recent years, accentuating the need for increased efforts to prevent and manage NIHL. This review will highlight the current research and future trends in noise pollution and its auditory impact in human beings.

\section{Pathophysiology of Noise-Induced Hearing Loss (NIHL)}

Besides age-related hearing loss, NIHL is the second most common form of hearing loss [10]. However, excess noise is one of the major preventable causes of hearing loss. The distinct pathology of NIHL is the loss of hair cells in the Organ of Corti. Since mammals including humans do not have the ability to regenerate hair cells, NIHL could be irreversible. Outer hair cells (OHCs) are more vulnerable to noise exposure than the inner hair cells. Similarly, the basal region of the organ of Corti is more susceptible to noise induced injury compared to the low-frequency apical region. The microscopic features of NIHL include the destruction of OHCs, floppy stereocilia, fusion and loss of stereocilia, loss of adjacent supporting cells, complete disruption of the organ of Corti, progressive Wallerian degeneration, and loss of primary auditory nerve fibers.

Significant achievements have been made in the understanding of the cellular and molecular mechanisms of NIHL which could be helpful in developing therapeutic strategies. Cochlear injury due to excessive noise can occur through mechanical and metabolic mechanisms both. Noise exposures of around 115-125 dB SPL, mechanical injury tends to dominate. However, in most circumstances, the level of noise exposure to the ear is $<115 \mathrm{~dB}$, and the damage is mainly metabolically driven [11]. A complex interplay of genetic and environmental factors leads to oxidative stress at the molecular level. Oxidative stress theory for noiseinduced cochlear injury asserts that acoustic overexposure causes production of reactive oxygen species (ROS) and reactive nitrogen species which oxidize cell membrane lipids, intracellular proteins, and DNA, production of proinflammatory cytokines interleukin-6 (IL-6) and tumour necrosis factor $\alpha$ (TNF-@), vasoactive lipid peroxidation products such as isoprostanes leading to reduced cochlear blood flow, disproportionate release of neurotransmitters such as glutamate, and increase in intracellular calcium happens. A vital element of cochlear microcirculation is the diameter of the spiral modiolar artery, a branch of the anterior inferior cerebellar artery, which joins the lateral cochlear wall to form the stria vascularis. The spiral modiolar artery has been demonstrated to constrict during and after exposure to noise [12]. Glutamate excitotoxicity, GSH depletion, excessive increases in intracellular calcium, and ischemia reperfusion can all lead to mitochondrial injury and have been implicated in noise injury to the cochlea. ${ }^{11}$ Mitochondrial membranes become permeable and release respiratory enzyme molecules (e.g., cytochrome c) that activate cell death effector proteins (e.g., caspases). Programmed cell death pathways involve calpain, caspases, and c-Jun N-terminal kinase Jun molecules. Cochlear antioxidant defences (antioxidant enzymes, heat shock proteins [HSPs], trophic factors, vitamins C and E, and glutathione [GSH]) gets exhausted. Both inflammatory and immune responses are key mechanisms in the cochlear defensive response to noise and if unregulated, contribute to inner ear damage and hearing loss. Transforming growth factor $\beta$ (TGF- $\beta$ ) is a key regulator of both of these responses, and high levels of this factor have been associated with cochlear injury in animal models [11].

Another new opportunity of research in this area has opened yet another dimension- that of genetic predilection to NIHL. Rick A Friedman identified the Nox3, which is almost exclusively expressed in the inner ear, as a key gene predisposing to NIHL [13]. An increased susceptibility to NIHL may rely on the SNPs of several other genes, including the groups of oxidative stress genes, $\mathrm{K}+$ ions recycling genes, monogenic deafness genes, as well as mitochondrial genes [14]. Another study by Kowalski, et al. identified one of the most interesting candidate genes CDH23 encoding Cadherin 23 which is a component of stereocilia tip links in patients with high susceptibility to noise confirming this CDH-23 genetic variant may modify the susceptibility to NIHL development in humans [15]. Cadherins, the calciumdependent proteins, hold cells anchored at adherens junctions to form tissues and organs. The cadherin of interest named otocadherin or $\mathrm{CDH} 23$ is localized to the stereocilia of the OHCs. Reduction of or missing otocadherin weakens the cell and allows stereocilia to be more easily physically harmed by loud sounds and by aging $[16,17]$. Exposure to severe noise can induce HSPs, and exposure to moderate intensity noise has been reported to confer protection against noise-induced damage to hearing. Some haplotypes of hsp70 genes could possibly be associated with a higher predisposition to NIHL [18]. In the inner ear, P2X2 receptors are supposed to regulate sound transduction and auditory neurotransmission, OHC electromotility, inner ear gap junctions, and $\mathrm{K}+$ recycling [19]. The absence or mutation of $\mathrm{P} 2 \mathrm{X} 2$ receptor increases vulnerability to NIHL. Apoptosis of cell is executed by a family of cysteine proteases called caspases [20]. X-linked inhibitor of apoptosis suppresses caspase- 3 activity and may decrease NIHL. Proteins of the Bcl2 family have been involved in regulating sensory cell survival and control of apoptotic pathways modulating neuronal cell death, including NIHL [21]. The Bcl2 family consists of a group of proapoptotic and antiapoptotic molecules that regulate caspase activation. The mechanisms of sensory hair cell degeneration in response to different ototoxic stimuli share a terminal common pathway: caspase activation. Inhibition of caspases delays or prevents hair cell death and may possibly preserve hearing/balance function. Inhibition of mitogen-activated protein kinases (MAPK) defends against noise-induced hair cell death [22]. BclxL plays an essential role in the prevention of sensory 
cell death following TTS levels of noise, and PTS exposure provokes the expression of Bak and with that, cell death [21]. Glutathione-S-transferase (GST) enzymes are vital for antioxidant protection. Genetic variability in GST enzymes upsurges vulnerability to damage from oxidation. It has been proposed that an person with a null genotype is more prone than his peers to develop NIHL when exposed to excessive noise [12]. There are also some genetic mutations that lessen predisposition to NIHL. A1555G or other mitochondrial variants in the MTRNR1 and MTTS1 have been postulated as factors that lessen susceptibility to NIHL [12]. The additional research on the genetic susceptibility to NIHL has focused on human 8-oxoG DNA glycosylase1 (hOGG1), a key enzyme in the human base excision repair pathway. This DNA repair has been demonstrated to be essential in maintaining hearing after noise exposure. The hOGG1 Cys/Cys genotype may have a lesser capacity for restoring 8-oxoG damage, and thus a higher vulnerability for NIHL [12].

Atoh1 (also known as math1) has been found to be essential for neurogenesis in the central and peripheral nervous system, and for the formation of several nonneural cell types. In the auditory system, Atoh1 gene plays a crucial role in hair cell differentiation during development. The hallmark of mechanosensory hair cells is stereocilia, where mechanical stimuli are transformed into electrical signals. While hair cells in lower vertebrates and the mammalian vestibular system can spontaneously redevelop lost stereocilia, mammalian cochlear hair cells do not retain this capability during the developmental process. Atoh1 as a gene has been found to be critical for promoting repair/ regeneration of stereocilia and maintaining injured hair cells in the adult mammal cochlea [23].

\section{Prevention measures and management of noise- induced hearing loss}

Around one-third of permanent hearing loss is preventable through proper hearing loss prevention strategies. Hearing safety equipment and environmental engineering solutions to lessen noise are critical components of the preventive strategy [11]. Hearing aids and cochlear implants are the currently available management strategies for hearing loss. Considering the oxidative stress hypothesis for NIHL, many different therapeutic approaches could be available for managing NIHL by augmenting the cellular oxidative stress defence pathways in the cochlea [24]. Antioxidants that have been studied so far include $\mathrm{N}$-acetylcysteine, magnesium, salicylate, vitamin E and Ebselen [25,26]. Glucocorticoid receptors are expressed in the human cochlea. Glucocorticoids, such as cortisol, modulate hearing sensitivity and have been shown to confer some protective effects [27]. Calcium channel blockers or agents that improve cochlear blood flow are other possible treatment modalities. TGF- $\beta$ is a key regulator of both inflammatory and immune responses. This has opened the exciting possibility of targeting TGF- $\beta$ as a therapeutic strategy for preventing or ameliorating NIHL [28]. TGF- b 1 peptidic inhibitors P17 and P144 just before or immediately after noise exposure significantly improved hearing thresholds and the degenerative changes in lateral wall structures [28]. Likewise, another study by Martínez-Vega, et al. shown that long-term omega-3 fatty acid supplementation prevents expression changes in homocysteine metabolism and ameliorates progressive hearing loss in mice [29]. AcetylL-carnitine, a mitochondrial metabolite, have been found to restore mitochondrial membrane integrity and lessen mitochondrial free radical formation, thereby preventing mitochondrial-induced apoptosis. Cytochrome c release from the injured mitochondria can be prevented by averting pore formation (e.g., overexpression of Bcl-2 or BclxL though a gene therapy vector) [11]. Reducing glutamate excitotoxicity include opposing the damaging ionic fluxes non-specifically through magnesium supplementation, or more specifically, through application of an antagonist of glutamate receptorassociated ionic channels. Maintaining, increasing, and reinstating cochlear GSH levels, a key intracellular antioxidant and an inhibitor of stress-induced apoptosis, has numerous potential benefits as a treatment strategy to decrease NIHL. GSH can counteract the damaging effects of glutamate excitotoxicity, mitochondrial damage, and excessive intracellular calcium fluxes. A wide variety of GSH or cysteine precursors and cell-permeable forms i.e. esters of GSH (e.g.; NAC, methionine, GSH esters, and thiazolidinerelated drugs, such as 2-oxothiazolidine-4-carboxylate) have been found to decrease NIHL in in vitro studies [11]. Other more specific apoptotic pathway inhibitors, for example, leupeptin, a calpain inhibitor, inhibiting steps in the MAPK signalling pathway of apoptosis has been found to be somewhat effective in reducing noise injury to the cochlea. In addition, specific programmed cell death pathway inhibitors such as round window membrane application of c-Jun antisense oligonucleotide therapy or D-JNKI-1 treatment may prove to be beneficial clinically [11]. The olivocochlear system may help protect against acoustic trauma. Sound conditioning requires moderate-level sound exposures on a daily basis and may help decrease damage from a subsequent high-level noise exposure.

The ability to begin auditory sensory cell regeneration through gene therapy to restore NIHL may be a possibility in future. Mammals have not been demonstrated to form new hair cells after damage in mature animals. This phenomenon may be explained by the occurrence of cell proliferation inhibitors found to be active in the cells of the mature mammalian cochlea. Nonetheless, it could be possible to supersede this block on proliferative regeneration using trophic factors alone or in combination with inhibitors of cell proliferation inhibitors, but only in future times. Genes important for the genesis of hair cells in mammals, such as Notch, Math 1, Brn 3.1 have also been discovered [11]. The possibility of regenerating stereocilia in the noise deafened guinea pig 
cochlea by inoculation of a viral vector carrying Atoh1, the gene critical for differentiation of hair cells, promoting repair or regeneration of stereocilia and maintaining injured hair cells in the adult mammal cochlea has also been explored. Atoh1-based gene therapy, hence, has the potential to reverse noise-induced hearing loss if the treatment is carried out before the hair cells die [23]. Researchers conducted at Massachusetts Eye and Ear and Harvard Medical School have shown that hair cells can be regenerated through a drug which inhibits the enzyme gamma secretase. This impedes a signal produced by a protein known as Notch on supporting cells. Supporting cells are stimulated to develop as new hair cells resulting in partial regaining of hearing in mouse ears which were damaged by noise trauma [30].

\section{Conclusion and recommendations}

Noise-induced hearing loss (NHIL) remains a major cause of deafness globally. Hearing loss caused either by exposure to occupational or environmental noise is highly prevalent and constitutes a major public health challenge requiring preventive and therapeutic strategies. Through this review, we highlight that auditory health effects of environmental noise are manifold, critical and, because of widespread exposure, very prevalent. These considerations emphasize the need to regulate and reduce environmental noise exposure (ideally at the source) and to implement exposure limits to alleviate negative health consequences of chronic exposure to environmental noise. Educational campaigns for both children and adults can encourage noise avoiding and noise-reducing behaviours, and therefore, mitigate the negative health consequences. Further, insights into the molecular mechanisms of noise-induced cochlear damage can result in the development of new treatment strategies that could make the cochlea more resilient to noise exposure as well as augment the healing of noise-injured cochleae. Further advances in deciphering the genetic predisposition for NIHL will enable early screening and assist in developing prevention and treatment strategies.

\section{References}

1. Hughes RW, Jones DM. Indispensable benefits, and unavoidable costs of unattended sound for cognitive functioning. Noise Health. 2003; 6: 63-76.

PubMed: https://pubmed.ncbi.nlm.nih.gov/14965454/

2. Basner M, Müller $U$, Elmenhorst EM. Single and combined effects of air, road, and rail traffic noise on sleep and recuperation. Sleep. 2011; 34: 11-23.

PubMed: https://pubmed.ncbi.nlm.nih.gov/21203365/

3. Stansfeld S, Haines M, Brown B. Noise, and health in the urban environment. Rev Environ Health. 2000; 15: 43-82.

PubMed: https://pubmed.ncbi.nlm.nih.gov/10939085/

4. Miedema HME, Oudshoorn CGM. Annoyance from transportation noise: relationships with exposure metrics DNL and DENL and their confidence intervals. Environ Health Perspect. 2001; 109: 409-416. PubMed: https://www.ncbi.nlm.nih.gov/pmc/articles/PMC1240282/
5. Muzet A. Environmental noise, sleep and health. Sleep Med Rev. 2007; 11: 135-142.

PubMed: https://pubmed.ncbi.nlm.nih.gov/17317241/

6. van Kempen $\mathrm{E}$, Babisch $\mathrm{W}$. The quantitative relationship between road traffic noise and hypertension: a meta-analysis. J Hypertens. 2012; 30: 1075-1086.

PubMed: https://pubmed.ncbi.nIm.nih.gov/22473017/

7. Sørensen M, Andersen ZJ, Nordsborg RB, et al. Road traffic noise and incident myocardial infarction: a prospective cohort study. PLoS One. 2012; 7: e39283.

PubMed: https://pubmed.ncbi.nlm.nih.gov/22745727/

8. Stansfeld SA, Matheson MP. Noise pollution: non-auditory effects on health. Br Med Bull. 2003; 68: 243-257.

PubMed: https://pubmed.ncbi.nlm.nih.gov/14757721/

9. Babisch W. Exposure to environmental noise: risks for health and the environment. Workshop on "sound level of motor vehicles". Directorate General for Internal Policies of the European Parliament;Brussels:2020. http://www.europarl.europa.eu/document/activities/cont/201205/2012 0524ATT45762/20120524ATT45762EN.pdf.

10. Konings A, Van Laer L, Van Camp G. Genetic studies on noiseinduced hearing loss: A review. Ear Hear. 2009; 30: 151-159. PubMed: https://pubmed.ncbi.nlm.nih.gov/19194285/

11. Kopke RD, Coleman JK, Liu J, Jackson RL, Van De Water TR. Mechanisms of noise-induced hearing loss and otoprotective strategies. In: Van De Water TR, Staecker H, eds. Otolaryngology: Basic Science and Clinical Review. New York: Thieme; 2006; 395-408.

12. Aviva Levihaiem. The Impact of Acoustic Trauma on the Ear. https://www.las.touro.edu/media/schools-and-colleges/landercollegefor-arts-and-sciences/biology/publications/fall-2015/Noise_Induced Hearing_Loss_levihaiem.pdf.

13. Lavinsky J, Crow AL, Pan C, Wang J, Aaron KA, et al. Genome-wide association study identifies nox 3 as a critical gene for susceptibility to noise-induced hearing loss. PLoS Genet. 2015; 11: e1005094. PubMed: https://pubmed.ncbi.nlm.nih.gov/25880434/

14. Sliwiniska-Kowalska M, Pawelczyk M, Kowalski TJ. Genetic factors in susceptibility to age- and noise-related hearing loss. Pol Merkur Lekarski. 2006; 21: 384-388.

PubMed: https://pubmed.ncbi.nIm.nih.gov/17205784/

15. Kowalski TJ, Pawelczyk M, Rajkowska E, Dudarewicz A, SliwinskaKowalska M. Genetic variants of $\mathrm{CDH} 23$ associated with noiseinduced hearing loss. Otol Neurotol. 2014; 35: 358-365. PubMed: https://pubmed.ncbi.nlm.nih.gov/24448297/

16. Davis RR, Kozel P, Erway LC. Genetic influences in individual susceptibility to noise: A review. Noise Health. 2003; 5: 19-28. PubMed: https://pubmed.ncbi.nlm.nih.gov/14558889/

17. Yang $M$, Tan $H$, Zheng JR, Wang $F$, Jiang $C$, et al. Association of cadherin $\mathrm{CDH} 23$ gene polymorphisms with noise induced hearing loss in Chinese workers. Wei Sheng Yan Jiu. 2006; 35: 19-22. PubMed: https://pubmed.ncbi.nlm.nih.gov/16598924/

18. Yang $\mathrm{M}$, Tan $\mathrm{H}$, Yang $\mathrm{Q}$, Wang $\mathrm{F}$, Yao $\mathrm{H}$, et al. Association of hsp70 polymorphisms with risk of noise-induced hearing loss in Chinese automobile workers. Cell Stress Chaperones. 2006; 11: 233-239. PubMed: https://www.ncbi.nlm.nih.gov/pmc/articles/PMC1576471/

19. Yan D, Zhu Y, Walsh T, Xie D, Yuan $\mathrm{H}$, et al. Mutation of the ATPgated P2X (2) receptor leads to progressive hearing loss and increased susceptibility to noise. Proc Natl Acad Sci U S A. 2013; 110: 2228-2233.

PubMed: https://pubmed.ncbi.nlm.nih.gov/23345450/

20. Wang J, Tymczyszyn N, Yu Z, Yin S, Bance M, Robertson GS. Overexpression of $\mathrm{X}$-linked inhibitor of apoptosis protein protects 
against noise-induced hearing loss in mice. Gene Ther. 2011; 18 : 560-568.

21. Yamashita D, Minami SB, Kanzaki S, Ogawa K, Miller JM. Bcl-2 genes regulate noise-induced hearing loss. J Neurosci Res. 2008; 86: 920-928.

PubMed: https://pubmed.ncbi.nlm.nih.gov/17943992/

22. Cheng AG, Cunningham LL, Rubel EW. Mechanisms of hair cell death and protection. Curr Opin Otolaryngol Head Neck Surg. 2005; 13: 343-348.

PubMed: https://pubmed.ncbi.nlm.nih.gov/16282762/

23. Yang SM, Chen W, Guo WW, Jia S, Sun JH, et al. Regeneration of stereocilia of hair cells by forced Atoh1 expression in the adult mammalian cochlea. PLoS One 2012; 7: e46355.

PubMed: https://pubmed.ncbi.nlm.nih.gov/23029493/

24. KopkeRD, ColemanJK,LiuJ,CampbellKC, RiffenburghRH.Candidate's thesis: Enhancing intrinsic cochlear stress defenses to reduce noise-induced hearing loss. Laryngoscope. 2002; 112: 1515-1532. PubMed: https://pubmed.ncbi.nlm.nih.gov/12352659/

25. Coleman JK, Kopke RD, Liu J, Ge X, Harper EA, et al. Pharmacological rescue of noise induced hearing loss using $\mathrm{N}$-acetylcysteine and acetyl-L-carnitine. Hear Res. 2007; 226: 104-113.

PubMed: https://pubmed.ncbi.nlm.nih.gov/17023129/
26. Sendowski I, Raffin F, Braillon-Cros A. Therapeutic efficacy of magnesium after acoustic trauma caused by gunshot noise in guinea pigs. Acta Otolaryngol. 2006; 126: 122-129.

PubMed: https://pubmed.ncbi.nlm.nih.gov/16428187

27. Canlon B, Meltser I, Johansson P, Tahera Y. Glucocorticoid receptors modulate auditory sensitivity to acoustic trauma. Hear Res. 2007; 226: 61-69.

PubMed: https://pubmed.ncbi.nlm.nih.gov/16843624/

28. Murillo-Cuesta S, Rodríguez-de la Rosa L, Contreras J, Celaya AM, Camarero $\mathrm{G}$, et al. Transforming growth factor $B 1$ inhibition protects from noise-induced hearing loss. Front Aging Neurosci. 2015; 7: 32. PubMed: https://pubmed.ncbi.nlm.nih.gov/25852546/

29. Martínez-Vega R, Partearroyo T, Vallecillo N, Varela-Moreiras G, Pajares MA, et al. Long-term omega- 3 fatty acid supplementation prevents expression changes in cochlear homocysteine metabolism and ameliorates progressive hearing loss in C57BL/6J mice. J Nutr Biochem. 2015; 26: 1424-1433.

PubMed: https://pubmed.ncbi.nlm.nih.gov/26321228/

30. Mizutari K, Fujioka M, Hosoya M, Bramhall N, Okano HJ, et al. Notch inhibition induces cochlear hair cell regeneration and recovery of hearing after acoustic trauma. Neuron. 2013; 77: 58-69. PubMed: https://pubmed.ncbi.nlm.nih.gov/23312516/ 